DOI: $10.15593 / 2224-9982 / 2016.47 .07$

УДК 533:535.37

\author{
Г.М. Жаркова, В.Н. Коврижина, А.П. Петров \\ Институт теоретической и прикладной механики им. С.А. Христиановича \\ Сибирского отделения РАН, Новосибирск, Россия
ИССЛЕДОВАНИЕ ВЛИЯНИЯ ОДНОМЕРНЫХ ФОТОННЫХ КРИСТАЛЛОВ НА СВОЙСТВА ЧУВСТВИТЕЛЬНОГО \\ К ДАВЛЕНИЮ ЛЮМИНОФОРА
}

\begin{abstract}
Обсуждаются свойства чувствительных к давлению пленочных покрытий на основе порфиринового комплекса платины PtOEP, которые используются для измерения распределения давления на поверхности обдуваемой модели. Их применение основано на физическом эфффекте тушения фосфоресценции люминофора кислородом. Для усиления эмиссии люминофора предложено вводить в состав покрытия хиральные жидкие кристаллы, проявляющие свойства одномерных фотонных кристаллов. Наличие фотонной запрещенной зоны (ФЗ3) кристаллов влияет на спектр фосфоресценции молекул люминофора. В случае совпадения спектра фоссроресценции исследуемого люминофора с концом ФЗЗ кристаллов интенсивность фоссфоресценции PtOEP резко возрастает. Разработаны смеси хиральных жидких кристаллов на основе эфиров холестерина, у которых конец запрещенной фотонной зоны совпадает с пиком фоссроресценции PtOEP. Исследованы спектральные и динамические характеристики пленочных покрытий на основе люминофора PtOEP, поливинилацетата и хиральных жидких кристаллов. Показано, что введение фотонных кристаллов в состав покрытия приводит к увеличению его чувствительности к давлению.
\end{abstract}

Ключевые слова: жидкие кристаллы, бароиндикаторное покрытие, фосфоресценция, люминофор, фотонные кристаллы.

\author{
G.M. Zharkova, V.N. Kovrizhina, A.P. Petrov
}

Khristianovich Institute of Theoretical and Applied Mechanics Siberian Branch of Russian Academy of Sciences, Novosibirsk, Russian Federation

\title{
EFFECT OF THE PHOTONE CRYSTALS ON THE PROPERTIES OF THE PRESSURE-SENSITIVE LUMINOPHOR
}

The paper discusses the properties of the pressure-sensitive films, based on the porphyrin platinum complex PtOER. Their use is based on the physical effect of the quenching of phosphorescence by oxygen. To enhance the luminophor emission it is proposed to introduce into the coating chiral liquid crystals, showing one-dimensional properties of photon crystals. When the phosphorescence spectrum of the phosphor coincides with the end of band gap of photon crystals the phosphorescence intensity increases rapidly. The mixtures of chiral liquid crystals on the basis of cholesterol esters in which the end of the photonic band gap coincides with the phosphorescence PtOER were developed. The spectral and dynamical characteristics of film based on PtOER, polyvinyl acetate and chiral liquid 
crystal were studied. It was shown that the introduction of the $\mathrm{ChLCl}$ in the film composition leads to an increase in its pressure sensitivity. crystals.

Keywords: liquid crystals, pressuary-sensitive coating, phosphorescence, luminophor, photonic

\section{Введение}

Оптические сенсоры давления на основе органических люминофоров широко применяются в различных областях науки и техники. Полные сведения о люминесцентных сенсорах давления, разработанных для аэродинамических экспериментов, можно найти в обзорах $[1,2]$. Их применение основано на физическом эффекте тушения люминесценции люминофора кислородом. Люминофоры, используемые в чувствительных к давлению покрытиях, должны обладать высоким квантовым выходом люминесценции, длительным временем жизни, большим сдвигом Стокса и хорошей фотостабильностью [3, 4]. Анализ используемых для этих целей люминофоров показал, что веществ, чьи максимумы возбуждения и излучения находятся в видимой области спектра, а свойства зависят только от давления и не зависят от температуры, ограниченное число.

Дальнейшее развитие люминесцентных сенсоров связано с синтезом новых стабильных индикаторов, имеющих высокие квантовые выходы. Это длительный и дорогой химический процесс. Другой путь искать способы улучшения характеристик уже известных люминофоров путем применения физических эффектов, способных изменять чувствительность и время отклика сенсора в заданном диапазоне концентраций кислорода. Один из возможных способов - применение фотонных кристаллов. Фотонные кристаллы (ФК) привлекают особое внимание при создании оптических сенсоров различного назначения. Фотонные кристаллы - это структурно организованные среды, диэлектрические свойства которых меняются периодически в одном, двух или трех измерениях [5]. Благодаря этому в ФК существуют фотонные запрещенные зоны (ФЗ3), в которых электромагнитные волны не могут распространяться, испытывая отражение от структуры. Модификация спектра ФК может быть реализована посредством управления анизотропией этих оптических материалов. К таким материалам относятся жидкие кристаллы (ЖК), обладающие сильной анизотропией диэлектрической проницаемости. Создание ФК-структур с включением ЖК оказывается перспективным для управления их оптическими свойст- 
вами. Проблемам управления свойствами фотонных кристаллов, сформированных с включением жидких кристаллов, посвящены многочисленные работы и обзоры [6-8]. В большей степени это связано с открытием лазерной генерации в хиральных жидких кристаллах (ХЖК), допированных красителями [9-14]. Теоретическое объяснение увеличения эмиссии красителя в таких средах рассматривалось в работе [7]. Кратко этот эффект можно описать следующим образом.

Хиральные жидкие кристаллы, обладающие спиральной структурой, являются одномерными ФК с фотонной запрещенной зоной (областью селективного рассеяния света) для света, распространяющегося вдоль оптической оси ХЖК с круговой поляризацией, совпадающей со знаком холестерической спирали [15]. Световые волны с противоположной круговой поляризацией проходят через ХЖК почти без изменения.

Наличие запрещенной зоны в ХЖК влияет на спектр фосфоресценции молекул гостя. В случае совпадения полосы фосфоресценции исследуемого красителя с полосой ФЗЗ ХЖК плотность фотонных состояний фосфоресцентной полосы резко увеличивается на краях ФЗ3. Интенсивность фосфоресценции, почти нулевая внутри запрещенной зоны, усиливается на границах и испытывает сильные колебания вблизи стоп-зоны.

Представляет интерес использование этого эффекта для модификации свойств люминофоров, традиционно применяемых в бароиндикаторных сенсорах путем внесения их в хиральную жидкокристаллическую среду.

Цель работы - исследование влияния фотонных жидких кристаллов на амплитудные и временные характеристики порфиринового комплекса платины в зависимости от давления.

\section{Техника эксперимента и материалы}

\section{Экспериментальная установка}

Для измерения характеристик люминесценции (интенсивности и времени тушения) исследуемых бароиндикаторных покрытий образец с нанесенным покрытием помещался в барокамеру, давление в которой понижалось форвакуумным насосом и контролировалось вакуумметром. Возбуждение люминофора в образце осуществлялось лучом 
лазера с длиной волны $\lambda=532$ нм, который через четвертьволновую и светоделительную пластинки направлялся на образец. Часть луча, отраженная от светоделительной пластинки, попадала на фотоэлектронный умножитель (ФЭУ 10) для контроля интенсивности лазера. Излучение люминесценции выделяется светофильтром КС-11 и объективом собирается на катоде ФЭУ. При измерении амплитудных и временных характеристик возбуждаемой фосфоресценции покрытия луч лазера прерывается модулятором, а сигналы с ФЭУ регистрируются двухлучевым осциллографом Tektronix 15. Схема измерительной установки подробно описана в работе [4].

Спектральные характеристики измерялись при отключенном модуляторе, а излучение, собираемое объективом, подавалось световодом на входную щель спектрофотометра «Колибри». Параметры затухания люминесценции исследуемых покрытий снимались с использованием излучения импульсного азотного лазера с длиной волны $\lambda=330 \mathrm{Hм}$ и длительностью 20 нс.

\section{Материаль}

В состав исследуемых образцов входили: люминофор, жидкие кристаллы, связующее вещество, растворитель. В качестве связующего (полимерной матрицы) использовался поливинилацетат (ПВА), люминофора - октаэтил порфирин платины (PtOEP), жидких кристаллов эфиры холестерина и ряда жирных кислот.

Образцы формировались двумя способами: напылением раствора или капсулированием ЖК + люминофор в полимерной матрице. Первый способ: смесь перечисленных соединений методом напыления наносится на стекло или лавсановую пленку. Однако этот способ не позволяет создать в образце совершенную планарную текстуру ЖК, которая должна обеспечивать периодическую фотонную структуру кристаллов. Второй способ: смесь люминофора и ЖК в определенном соотношении вводится в (12\%-ный) раствор поливинилацетата и затем наносится на стеклянную подложку. Выбор полимера определялся тем, что ЖК находятся в этой полимерной матрице в виде капель и образуют так называемую биполярную текстуру, оптические свойства которой близки к свойствам планарной текстуры ЖК [16], для которой характерно наличие ФЗ3. 
На рис. 1 представлены спектры пропускания и фосфоресценции люминофора. Видно, что люминофор имеет одну из полос поглощения, совпадающую с длиной волны зеленого лазера, что позволяет эффективно возбуждать его на этой длине волны и получать максимальное отношение сигнал/шум.

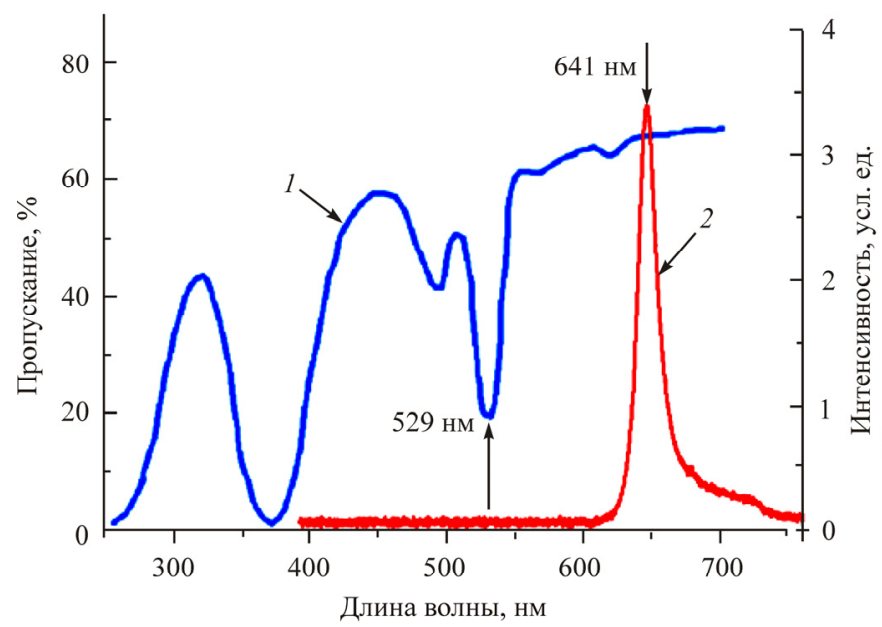

Рис. 1. Спектры пропускания (1) и фосфоресценции (2) PtOEP

В работе [4] отмечено, что интенсивность излучения PtOEР снижается, а время жизни уменьшается при более высоких концентрациях его в покрытии. Этот эффект наблюдался при концентрациях выше 1 г люминофора на 120 г полимера. Исследование концентрационной зависимости интенсивности фосфоресценции от содержания люминофора в полимерной матрице (ПВА) показало, что наибольший сигнал получается при соотношении люминофор : ПВА 1:120 (рис. 2). Далее все исследуемые образцы содержали это количество люминофора и возбуждались излучением лазера мощностью 1 мВт.

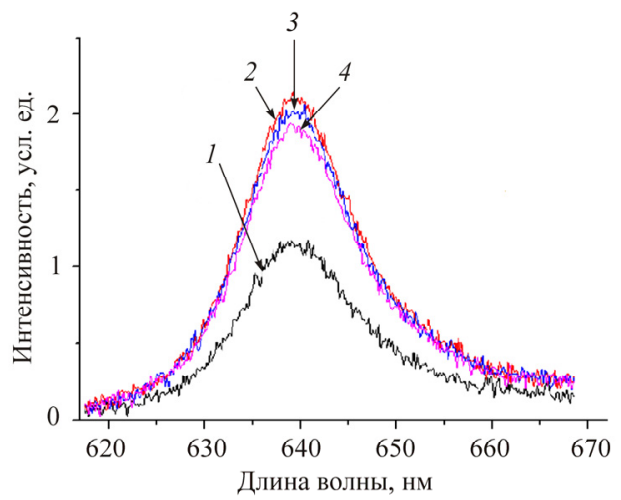

Рис. 2. Зависимость интенсивности фосфоресценции от соотношения концентрации люминофора и концетрации полимера в пленке: 1 - 240; 2 - 120; 3 - 85; $4-60$ 


\section{Экспериментальные результаты и их обсуждение}

Для формирования жидкокристаллической среды, характеризующейся периодической структурой и обладающей свойствами фотонного кристалла, применялись смеси следующих эфиров холестерина: пеларгонат, валерат, хлорид.

Основными задачами были разработка и исследование ЖК-составов, фотонная запрещенная зона которых была бы в области, удаленной от пика фосфоресценции люминофора. Исследовался ряд составов на основе смеси эфиров (пеларгонат - 70 и валерат -30 вес. \%), в которую дополнительно вводился холестерилхлорид (Хл) в количестве от 23 до 30 вес. \%.

Меняя соотношение компонентов, можно изменять ширину ФЗ3 так, чтобы полоса люминесценции люминофора приходилась на конец Ф33 жидкого кристалла. Таким условиям удовлетворяют две смеси жидких кристаллов с содержанием холестерилхлорида 20 и 30 вес. \% (рис. 3). Обозначение исследованных смесей Хл20 и Хл30.

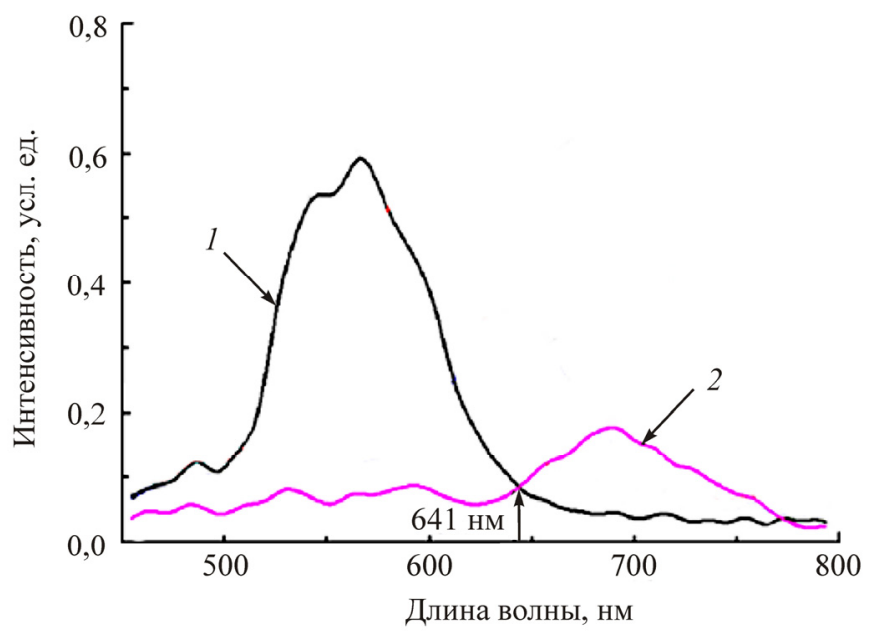

Рис. 3. Спектры отражения покрытий с разными ЖК при освещении ксеноновой лампой: 1 - Хл20; 2 - Хл30

Положение максимума фосфоресценции люминофора отмечено стрелкой на оси длин волн. Видно, что оно приходится на границу длинноволновой части пика отражения состава Хл20 и коротковолновой - состава Хл30. 
Дальнейшие исследования проводились с этими двумя смесями ЖК, которые добавлялись в полимер при соотношении ЖК : ПВА - 1:1 и люминофор : ПВА - 1:120. На рис. 4 показаны зависимости амлитудных и динамических характеристик исследуемых покрытий от состава ЖК и давления. Сравнивались отношения интенсивностей фосфоресценции и постоянных затухания при разных давлениях. Для образцов, не содержащих жидкие кристаллы, отношение интенсивности фосфоресценции при давлении $10^{2}$ Па к интенсивности при давлении $10^{5}$ Па составляет около 1,6. Эти же отношения для образцов, содержащих жидкие кристаллы ЖК20 и ЖК 30 , равны 5 и 4,8 соответственно. Это увеличивает чувствительность покрытия к давлению в три раза. Значения постоянной затухания определялось как время, за которое величина сигнала снижалась в $e$ раз от максимального значения. В образцах с использованием ЖК постоянные затухания уменьшаются: при атмосферном давлении и в вакууме они соответственно равны 230-155 мкс. Времена затухания образцов, не содержащих ЖК, 250 и 190 мкс.

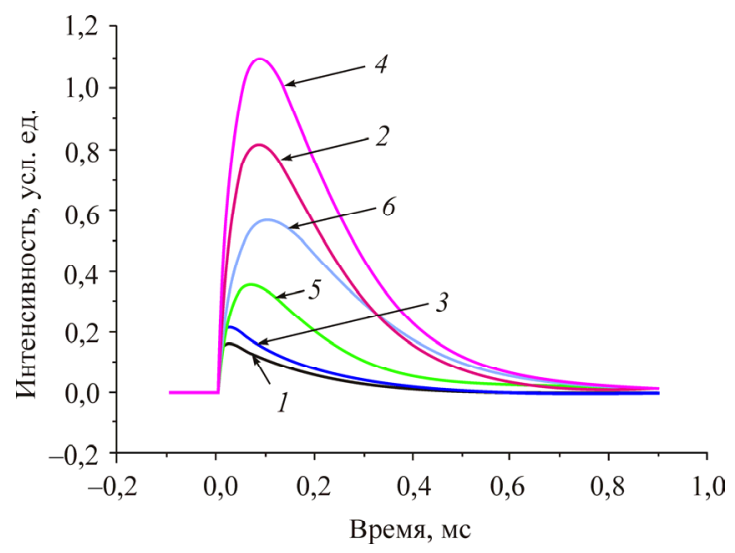

Рис. 4. Влияние состава ЖК и давления на кинетику затухания фосфоресценции: 1 - ЖК20 $\left(10^{5}\right.$ Па); 2 - ЖК20 (10² Па); 3 - ЖК30 (10 Па); 4 - ЖК30 (10² Па); 5 - Pt в ПВА $\left(10^{5} \Pi а\right) ; 6-\mathrm{Pt}$ в ПВА $\left(10^{2} \Pi а\right)$

Поскольку коэффициент рефракции ЖК зависит от температуры, необходимо было измерить зависимость интенсивности фосфоресценции PtOEР в полимере от температуры (рис. 5). С увеличением температуры интенсивность фосфоресценции РtOEP падает. Следовательно, 
необходимо производить корректировку данных по давлению, полученных с помощью этих покрытий, с учетом температуры.

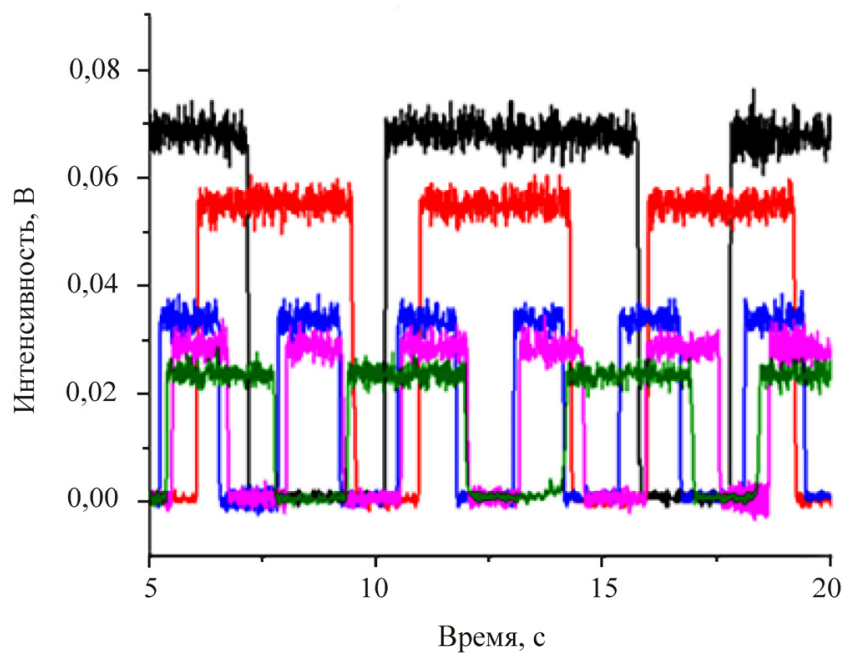

Рис. 5. Зависимость интенсивности фосфоресценции покрытия, содержащего смесь ЖК20,

от температуры: $-20^{\circ} ;-30^{\circ} ;-40^{\circ} ;-50^{\circ} ;-60^{\circ}$

\section{Заключение}

Исследовано влияние добавки хиральных жидких кристаллов, обладающих свойствами фотонного кристалла, на чувствительность бароиндикаторных пленок на основе порфиринового комплекса платины. Показано, что чувствительность пленки в области давлений от $10^{5}$ до $10^{2}$ Па увеличивается в три раза. Чувствительность к давлению смесей, для которых спектр фосфоресценции соответствует коротковолновой и длинноволновой границам запрещенной зоны жидких кристаллов, практически не отличается. Использование поливинилацетата в качестве полимерной матрицы отражается на временных и температурных характеристиках покрытия, поэтому данные по давлению, полученные с помощью покрытий, необходимо корректировать с учетом температуры.

Работа выполнена в рамках государственного задания № 03232014-0011. 


\section{Библиографический список}

1. Mosharov V.E., Radchenko V.N., Fonov S. Luminescent pressure sensors in aerodynamic experiment / Central Aerohydrodynamic Institute CWA International Corporation, 1997. - $151 \mathrm{p}$.

2. Liu T., Sullivan J.P. Pressure and temperature sensitive paints. Springer, 2005. $-328 \mathrm{p}$.

3. Применение эксимерных лазеров для исследования кинетики затухания люминесценции бароиндикаторных покрытий / Г.М. Жаркова, А.Н. Малов, В.М. Хачатурян, А.А. Лопаткина // Автометрия. 2003. - T. 39, № 5. - С. 112-117.

4. Исследование тушения люминесценции порфириновых комплексов платины в полимерной матрице / Г.М. Жаркова, А.П. Петров, А.Г. Бессонов, А.С. Семейкин, В.В. Быкова, Н.В. Усольцева // Жидкие кристаллы и их практическое использование. - 2012. - № 1(39). C. 31-41.

5. Joannopoulos J., Meade R., Winn J. Photonic crystals. - Princeton Univercity, 1995. - $137 \mathrm{p}$.

6. Chiral luminescent compounds as a perspective for cholesteric liquid crystal lasers / G. Petriashvili, G. Chilaya, M. Ariosto Matranga [et al.] // Optical Materials. - 2009. - № 31. - P. 1693-1696.

7. Невская Г.Е., Палто С.П., Томилин М.Г. Микролазеры на жидких кристаллах // Оптический журнал. - 2010. - Т. 77, № 8. - С. 13-31.

8. Шабанов В.Ф., Ветров С.Я., Шабанов А.В. Оптика реальных фотонных кристаллов. Жидкокристаллические дефекты, неоднородности. - Новосибирск: Изд-во СО РАН, 2005. - 209 с.

9. Kogelnic H., Shank C.V. Stimulated emission in a periodic structure // Appl. Phys. - 1972. - Vol. 18. - P. 152-154.

10. Study of optical second harmonic generation in ferroelectric liquid crystal / A.N. Vtyurin, V.P. Ermakov, B.I. Ostrovskii, V.F. Shabanov // Phys. Stat. Sol. (b). - 1981. - Vol. 107, № 1. - P. 397-402.

11. Самодифракция лазерного излучения на жидкокристаллических структурах / А.А. Ковалев, Г.Л. Некрасов, В.А. Пилипович [и др.] // Письма в ЖТФ. - 1979. - Т. 5, № 3. - С. 159-161.

12. Генерация второй оптической гармоники в жидких кристаллах; симметрия молекул и макроскопическая нелинейность / С.М. Аракелян, Г.Л. Григорян, С.Ц. Персисян [и др.] // Письма в ЖЭТФ. 1978. - T. 28, № 8. - C. 202-206. 
13. Bermel P.A., Warner M. Photonic band structure of cholecteric elastomers // Phys. Rev. E. - 2003. - Vol. 65. - P. 056614-056623.

14. Busch R., John S. Liquid crystal photonic band gap materials: the tunable electromagnetic vacuum // Phys. Rev. Lett. - 1999. - Vol. 83, № 5. P. 967-970.

15. Кац Е.И. Оптические свойства холестерических жидких кристаллов // ЖЭТФ. - 1970. - Т. 59. - С. 1854-1862.

16. Жаркова Г.М., Сонин А.С. Жидкокристаллические композиты. - Новосибирск: Наука, 1994. - 214 с.

\section{Referenses}

1. Mosharov V.E., Radchenko V.N., Fonov S. Luminescent pressure sensors in aerodynamic experiment. Central Aerohydrodynamic Institute CWA International Corporation, 1997. $151 \mathrm{p}$.

2. Liu T., Sullivan J.P. Pressure and temperature sensitive paints. Springer, 2005. $328 \mathrm{p}$.

3. Zharkova G.M., Malov A.N., Khachaturyan V.M., Lopatkina A.A. Primenenie eksimernykh lazerov dlya issledovaniya kinetiki zatukhaniya lyuminestsentsii baroindikatornykh pokrytiy [The application of excimer lasers for the study of the quenching luminescence kinetics of pressuarysensitive coatings]. Avtometriya, 2003, vol. 39, no. 5, pp. 112-117.

4. Zharkova G.M., Petrov A.P., Bessonov A.G., Semeykin A.S., Bykova V.V., Usoltseva N.V. Issledovanie tusheniya lyuminestsentsii porfirinovykh kompleksov platiny v polimernoy matritse [Study of the luminescence quenching of the luminescence of platinum porphyrin complexes in the polymer matrix]. Zhidkie kristally $i$ ikh prakticheskoe ispolzovanie, 2012, no. 1(39), pp. 31-41.

5. Joannopoulos J., Meade R., Winn J. Photonic crystals. Princeton Univercity, 1995. $137 \mathrm{p}$.

6. Petriashvili G., Chilaya G., Ariosto Matranga M. [et al.]. Chiral luminescent compounds as a perspective for cholesteric liquid crystal lasers. Optical Materials, 2009, no, 31, pp. 1693-1696.

7. Nevskaya G.E., Palto S.P., Tomilin M.G., Mikrolazery na zhidkikh kristallakh [Microlaser liquid crystals]. Opticheskiy zhurnal, 2010, vol. 77, no. 8, pp. 13-31.

8. Shabanov V.F., Vetrov S.Ya., Shabanov A.V. Optika realnykh fotonnykh kristallov. Zhidkokristallicheskie defekty, neodnorodnosti 
[Optics of real photonic crystals. Liquid crystal defects and heterogeneity]. Novosibirsk: Sibirskoe otdelenie Rossiyskoy akademii nauk, 2005. 209 p.

9. Kogelnic H., Shank C.V. Stimulated emission in a periodic structure. Appl. Phys., 1972, vol. 18, pp. 152-154.

10. Vtyurin A.N., Ermakov V.P., Ostrovskii B.I., Shabanov V.F. Study of optical second harmonic generation in ferroelectric liquid crystal. Phys. Stat.Sol. (b), 1981, vol. 107, no, 1, pp. 397-402.

11. Kovalev A.A., Nekrasov G.L., Pilipovich V.A. [et al.]. Samodifraktsiya lazernogo izlucheniya na zhidkokristallicheskikh strukturakh [Selfdiffraction of laser radiation on liquid-crystal structures]. Pisma $v$ zhurnal tekhnicheskoy fiziki, 1979, vol. 5, no. 3, pp. 159-161.

12. Arakelyan S.M., Grigoryan G.L., Persisyan S.Ts. [et al.]. Generatsiya vtoroy opticheskoy garmoniki $\mathrm{v}$ zhidkikh kristallakh; simmetriya molekul i makroskopicheskaya nelineynost [Generation of second optical harmonics in liquid crystals; symmetry of molecules and macroscopic nonlinearity]. Pisma $v$ zhurnal eksperimentalnoy $i$ teoreticheskoy fiziki, 1978, vol. 28, no. 8, pp. 202-206.

13. Bermel P.A., Warner M. Photonic band structure of cholecteric elastomers // Phys. Rev. E., 2003, vol. 65, pp. 056614-056623.

14. Busch R., John S. Liquid crystal photonic band gap materials: the tunable electromagnetic vacuum // Phys. Rev. Lett, 1999, vol. 83, no. 5, pp. 967-970.

15. Kats E.I. Optical properties of cholesteric liquid crystals. Sov. Phys. JETP, 1971, no. 32(5), pp. 1004-1007.

16. Zharkova G.M., Sonin A.S. Zhidkokristallicheskie kompozity [Liquid-crystal composites]. Novosibirsk: Nauka, 1994. 214 p.

\section{Об авторах}

Жаркова Галина Михайловна (Новосибирск, Россия) - доктор технических наук, профессор, главный научный сотрудник Института теоретической и прикладной механики им. С.А. Христиановича СО PAH (630090, г. Новосибирск, ул. Институтская, д. 4/1, e-mail: zharkova@itam.nsc.ru).

Коврижина Валентина Николаевна (Новосибирск, Россия) кандидат технических наук, старший научный сотрудник Института теоретической и прикладной механики им. С.А. Христиановича СО 
РАН (630090, г. Новосибирск, ул. Институтская, д. 4/1, e-mail: kovrizh@itam.nsc.ru).

Петров Александр Павлович (Новосибирск, Россия) - кандидат технических наук, старший научный сотрудник Института теоретической и прикладной механики им. С.А. Христиановича СО РАН (630090, г. Новосибирск, ул. Институтская, д. 4/1, e-mail: petrov@ itam.nsc.ru).

\section{About the authors}

Galina M. Zharkova (Novosibirsk, Russian Federation) - Doctor of Technical Sciences, Professor, Head Researcher, Khristianovich Institute of Theoretical and Applied Mechanics, Siberian Branch of Russian Academy of Sciences (4/1, Institutskya st., Novosibirsk, 630090, Russian Federation, e-mail: zharkova@itam.nsc.ru).

Valentina N. Kovrizhina (Novosibirsk, Russian Federation) - Ph. D. in Technical Sciences, Senior Researcher, Khristianovich Institute of Theoretical and Applied Mechanics, Siberian Branch of Russian Academy of Sciences (4/1, Institutskya st., Novosibirsk, 630090, Russian Federation, e-mail: kovrizh@itam.nsc.ru).

Aleksandr P. Petrov (Novosibirsk, Russian Federation) - Ph. D. in Technical Sciences, Senior Researcher, Khristianovich Institute of Theoretical and Applied Mechanics, Siberian Branch of Russian Academy of Sciences (4/1, Institutskya st., Novosibirsk, 630090, Russian Federation, e-mail: petrov@itam.nsc.ru).

Получено 09.08.2016 